\title{
THE SIXTH REGULAR MEETING OF THE SOUTHWESTERN SECTION.
}

The sixth regular meeting of the Southwestern Section of the Society was held at the University of Kansas, Lawrence, Kansas, on Saturday, November 30, 1912. About thirty persons were present including the following nineteen members of the Society:

Professor C. H. Ashton, Dr. Elizabeth R. Bennett, Professor W. C. Brenke, Professor E. W. Davis, Dr. Otto Dunkel, Professor E. P. R. Duval, Professor R. R. Fleet, Professor W. H. Garrett, Professor W. A. Harshbarger, Professor E. R. Hedrick, Dr. S. Lefschetz, Miss Hazel H. MacGregor, Professor U. G. Mitchell, Dr. Mary W. Newson, Professor S. W. Reaves, Professor W. H. Roever, Professor G. H. Scott, Professor J. N. Van der Vries, Professor Marion B. White.

The morning session was held at $10 \mathrm{~A}$. M. and the afternoon session at 2:30 P. M. Professor Van der Vries presided. It was decided to hold the next meeting of the section at Columbia, Missouri, on November 29, 1913, and the following programme committee was elected: Professors Hedrick (chairman), Brenke, and Kellogg (secretary).

The members present were the guests at a smoker and reception given on the evening before the meeting at the Alpha Tau Omega chapter house.

The following papers were presented at this meeting:

(1) Professor W. H. Roever: "The design and theory of a mechanism for illustrating certain systems of lines of force and stream lines."

(2) Professor S. W. Reaves: "On the projective differential geometry of plane anharmonic curves."

(3) Dr. E. R. BenNetT: "Transitive groups of degree 107."

(4) Dr. E. R. Bennfit: "The order of the product of two substitutions."

(5) Professor ARnold Eмch: "On closed continuous curves."

(6) Professor R. D. Carmichael: "On the theory of relativity: mass, energy, gravitation; philosophical aspects."

(7) Professor Florian Cajori: "Historical note on the graphic representation of imaginaries before the time of Wessel." 
(8) Dr. S. LeFschetz: "Geometry on ruled surfaces."

(9) Dr. E. L. Dodd: "The error-risk of certain functions of the measurements."

(10) Dr. T. H. Gronwall: "On the Riemann zeta function (first paper)."

(11) Dr. T. H. Gronwall: "On the Riemann zeta function (second paper)."

(12) Dr. T. H. Gronwall: "On Dirichlet's series corresponding to complex characters."

(13) Dr. T. H. Gronwall: "On Picard's theorem."

(14) Professor E. R. HedRICK: "Direct definition of the logarithmic derivative."

(15) Professor Louis INGoLD: "Note on systems of integral equations."

In the absence of the authors, the papers of Professor Emch, Professor Carmichael, Dr. Dodd, and Dr. Gronwall were read by title, the paper of Professor Cajori was presented by Professor Mitchell, and the paper of Professor Ingold by Professor Hedrick. Abstracts of the papers follow below.

1. At the Chicago meeting (April 5-6, 1912) Professor Roever exhibited a mechanism for illustrating certain systems of lines of force. This consisted essentially of two wheels with radial spokes which could be made to rotate in nearly coincident planes and thus render visible the loci of the intersections of the spokes (Bulletin, volume 18, page 435). Professor Roever now shows that not only for radial spokes but also for spokes in the form of logarithmic spirals, the mechanism illustrates lines of force. By having several pairs of wheels with different systems of logarithmic spirals (in particular, of radial straight lines) it is possible, in virtue of the device for obtaining different angular velocity ratios, to obtain a great number of different systems of curves, each of which may be regarded as lines of force or stream lines in five or six different branches of mathematical physics. It is possible, by making time exposures, to obtain well-defined photographs of these systems of curves. This possibility adds to the usefulness of the mechanism. The mechanism and photographs of some of the systems of curves were exhibited.

2. In this paper Professor Reaves applies the theory of plane curves as developed by Halphen and Wilczynski to a 
study of certain projective differential properties of the anharmonic curves

$$
y=x^{\lambda}, \quad y=e^{x}, \quad \text { and } r=e^{m \theta} .
$$

For each of these curves are found the osculating conic at an arbitrary point and the locus of its center; the Halphen point and its locus; the point in which the tangent meets the straight line on which lie the three points of inflection of the eight-pointic nodal cubic of the point of contact, and the locus of this point. For each curve these loci are found to be curves of the same type and having the same invariant triangle as the curve from which they are derived. The osculating conic of the logarithmic spiral is studied in some detail and a construction is given for its center and axes.

3. Transitive groups of degree $p=2 q+1, p$ and $q$ being prime numbers, have been considered especially by Mathieu, de Séguier, and Miller and all groups of this type, $p<107$, have been determined. Professor Miller's method for the determination of these groups is applied by Miss Bennett to the determination of the transitive groups of degree 107. It is shown that only the six well-known transitive groups of this degree exist.

4. Miss Bennett determined the order of the product of two circular substitutions having common elements when the arrangement of the common elements satisfied certain conditions.

5. In a paper soon to be published, Professor Emch shows that in every closed continuous curve at least one square may be inscribed. The object of the present communication is the solution of the inverse problem: to find the parametric equations of all continuous curves through the vertices of any square in the plane.

Designating by $t$ a parameter, the equations of such a curve may always be written in the form

$$
\begin{aligned}
x=p+\frac{a}{2} \sqrt{2} \cos ( & \left.\frac{2 \pi t}{\omega}+\theta\right) \\
& +\prod_{k=0}^{3} \sin \left\{\frac{2 \pi t}{\omega}-(2 k+1) \frac{\omega}{8}\right\} F(t),
\end{aligned}
$$




$$
\begin{aligned}
y=q+ & \frac{a}{2} \sqrt{2} \sin \left(\frac{2 \pi t}{\omega}+\theta\right) \\
& +\prod_{k=0}^{3} \sin \left\{\frac{2 \pi t}{\omega}-(2 k+1) \frac{\omega}{8}\right\} G(t),
\end{aligned}
$$

where $F(t)$ and $G(t)$ are single valued continuous functions of $t$. If these are periodic (with the same period $\omega$ ), the curve represented by (1) is a closed curve. Every closed continuous curve through the vertices of a square may therefore be represented in the form (1).

If it is possible to show that the parametric equations of any closed curve

$$
x=\phi\left(t^{\prime}\right), \quad y=\psi\left(t^{\prime}\right),
$$

where $\phi\left(t^{\prime}\right)$ and $\psi\left(t^{\prime}\right)$ are any continuous coperiodic functions of $t^{\prime}$, by proper transformation of $t^{\prime}$ into $t$, can be reduced to (1), then every closed curve admits of at least one inscribed square.

6. Professor Carmichael's paper on relativity falls into two parts. The first part deals with the theory of mass, energy and gravitation. The characteristic conclusions of the theory of relativity are derived in a simple manner. These are then applied to the theory of the Kaufmann and Bucherer experiments concerning the ratio of electric charge to the mass of a moving charged body. There is then a discussion of the bearing of these experiments on the general question of the experimental proof of the theory of relativity. The second part of the paper contains a discussion of some philosophical questions connected with that part of the theory of relativity which is developed in this paper and in an earlier one by Professor Carmichael (see Physical Review, September, 1912, pages 153-176).

7. Professor Cajori refers to a letter of Wallis to Collins May 6, 1673, which gives Wallis's earliest attempts at visualization of imaginaries. There is ground for suspecting that the Wessel-Argand diagram was known earlier to Euler, Walmesley, and others, and that Gauss received suggestions from Euler. The most important contribution before Wessel is the diagram of W. J. G. Karsten, 1768, which exhibits the infinitely many logarithms of real and complex numbers. 
8. In this paper Dr. Lefschetz gives two proofs of a formula given by W. E. Story in 1883, applying to scrolls of any order $\mu$, for the number of intersections of curves of order $a, b$ meeting the generators in $\alpha$ and $\beta$ points. One of the proofs is based on a previous work of Severi, and the other, of an elementary character, on the theory of elimination.

9. Dr. Dodd uses the conception of the error-risk* to draw comparisons between the arithmetic mean and certain functions of the measurements, - the error-risk being a species of probable value. The following theorems are closely related to the theorems presented in a paper at the recent summer meeting.

The Gaussian Law [G. L.] is postulated in this form: viz., that the probability that the error $x$ of the measurement $m$ will lie in the interval $\left(x^{\prime}, x^{\prime \prime}\right)$ is given by

where $x^{\prime}<x^{\prime \prime}$.

$$
\frac{h}{1 \cdot \frac{\pi}{\pi}} \int_{x^{\prime}}^{x^{\prime \prime}} e^{-h^{2} x^{2}} d x
$$

The "true value" is designated by $a$, and the arithmetic mean of $n$ measurements by $M_{n}$, or simply $M$.

Theorem. Under the G. L., the probable value of the square of the error of $M$ is greater than that of $B M$, if $B$ is any constant such that

$$
1-\frac{2}{2 n h^{2} a^{2}+1}<B<1 \text {. }
$$

Usually $h a$ is very large. But, if not, then from the standpoint of the " mean error-risk," it is useless to make any measurements at all-with the purpose of taking the arithmetic mean-unless a sufficient number, $n$, are made so that

$$
2 n h^{2} a^{2} \geqq 1 \text {. }
$$

For, if (2) is not satisfied, the risk in accepting zero as the value of the unknown is less than that in accepting $M$.

A somewhat similar theorem is valid, involving the absolute value of the error of $M$, and another involving any even power of the error of $M$.

Now let

$$
M_{n}^{\prime}=+\sqrt{\frac{m_{1}^{2}+m_{2}^{2}+\cdots+m_{n}{ }^{2}}{n}},
$$

* "Das Fehlerrisiko," Czuber, Wahrscheinlichkeitsrechnung, I, p. 266. 
in parti ular,

$$
M_{2}^{\prime}=+i \sqrt{\frac{m_{1}^{2}+m_{2}^{2}}{2}} .
$$

Theorem. Under the G. L., the probable value of the square of the error of $M_{2}^{\prime}$ is less than that of $M_{2}$, if $a>0$, and $h$ is sufficiently great.

Theorem. Under the G. L., the probable value of the square of the error of $M_{n}^{\prime}$ is greater than that of $M_{n}$ if $n>3$, and $h$ is sufficiently great.

By a weighted mean $W$ is meant $p_{1} m_{1}+p_{2} m_{2}+\cdots+p_{n} m_{n}$, where the $p$ 's are constants whose sum is unity; and by an absolute error an error taken positively.

Theorem. If the measurements are subject to the G. L. with measures of precision $h_{1}, h_{2}, \cdots, h_{n}$, respectively, then the probable value of any given positive power of the absolute error of $W$ is least when $p_{i}=h_{i}{ }^{2} / \Sigma h^{2}$. In particular, if the $h$ 's are all equal, this $W$ with the least error-risk is $M$.

10. Let $s=\sigma+t i$ be a complex variable; for $\sigma>1$, the Riemann zeta function is defined by the Dirichlet series

$$
\zeta(s)=\sum_{n=1}^{\infty} \frac{1}{n^{s}},
$$

which ceases to converge for $\sigma=1$. On this line, and for $|t|$ sufficiently large, the following upper and lower limitations for the zeta function were found by Mellin and Landau:

$$
\begin{gathered}
|\zeta(1+t i)|<\log |t|+c_{1}, \\
\frac{1}{|\zeta(1+t i)|}<c_{2} \log |t| \cdot \log \log |t|,
\end{gathered}
$$

where $c_{1}$ and $c_{2}$ are positive constants.

In the present paper, Dr. Gronwall proves, by relatively elementary means, the closer limitations

$$
\begin{gathered}
|\zeta(1+t i)|<\frac{3}{4} \log |t|+c_{1}, \\
\frac{1}{|\zeta(1+t i)|}<c_{2} \log |t| \cdot(\log \log |t|)^{\frac{3}{3}} .
\end{gathered}
$$


11. In continuation of the investigations of the preceding paper, and using some of the less elementary properties of the zeros of the zeta function, Dr. Gronwall finds that

$$
\begin{aligned}
& \frac{1}{|\zeta(1+t i)|}<c_{2} \log |t|, \\
& \left|\frac{\zeta^{\prime}(1+t i)}{\zeta(1+t i)}\right|<c_{3} \log |t| .
\end{aligned}
$$

12. In showing that any arithmetical progression $k x+l$, where $k$ and $l$ are relative primes, contains an infinity of prime numbers, Dirichlet introduced the series

$$
L_{\kappa}(s)=\sum_{n=1}^{\infty} \frac{\mathrm{X}_{\kappa}(n)}{n^{s}},
$$

where $\mathrm{X}_{\kappa}(n)$ is a certain $k$ th root of unity called the character of $n$. The main point in Dirichlet's investigation consisted in showing that $L_{k}(1) \neq 0$. For any complex character, Landau has recently obtained the limitation

$$
\frac{1}{\left|L_{\kappa}(1)\right|}<c \cdot(\log k)^{5}
$$

where $c$ is independent of $k$ and $l$. tion

In the present paper, Dr. Gronwall derives the closer limita-

$$
\frac{1}{\left|L_{\kappa}(1)\right|}<c \cdot \log k \cdot(\log \log k)^{\frac{3}{3}} .
$$

13. According to Landau's extension of Picard's theorem, there corresponds to any given power series

$$
a_{0}+a_{1} x+a_{2} x^{2}+\cdots
$$

a minimum radius $\varphi\left(a_{0}, a_{1}\right)$, depending on the first two coefficients only, and such that inside or on the circle $|x|=\varphi\left(a_{0}, a_{1}\right)$ the series either assumes one of the values 0 and 1 , or has a singular point. The exact expression for this radius was obtained by Carathéodory in terms of the modular function, while the ordinary elementary methods for proving the theorem in question only give an upper limit for the radius of a much higher order of magnitude. 
In the present paper, Dr. Gronwall obtains, by quite elementary considerations, upper and lower limits for this radius, each of which is of the same order of magnitude as the exact expression.

14. In this paper Professor Hedrick suggests several possible direct definitions of the logarithmic derivative, and discusses the effect of each of them upon certain fundamental theorems and upon the existence of the concept at points where the given function vanishes.

15. In this note Professor Ingold gives the general solution for $\varphi$ of the system of equations

$$
\int_{\alpha}^{\beta} a_{i}(x) \varphi(x) d x=b_{i}
$$

both for the case $i=1,2, \cdots, n$ and the case $i=1,2, \cdots, \infty$.

The result is there used to obtain a solution of the equation

$$
f(s)=\int_{a}^{\beta} K(s, t) \varphi(t) d t
$$

when the kernel can be expressed as a uniformly convergent series

$$
K(s, t)=\Sigma a_{i}(s) u_{i}(t)
$$

and $f$ may be written as a convergent series

$$
f(s)=\Sigma c_{i} a_{i}(s) .
$$

Neither of the systems $a_{i}(s), u_{i}(s)$ is assumed to be orthogonal, or to be closed; see also Picard, Rendiconti del Circolo Matematico, volume 29.

JoHN N. VAN DER VRIES, Secretary (pro tempore) of the Section. 